\title{
Spatial and Temporal Properties of Visual Responses in the Thalamus of the Developing Ferret
}

\author{
Colin J. Akerman, Matthew S. Grubb, and Ian D. Thompson \\ University Laboratory of Physiology, Oxford University, Oxford OX1 3PT, United Kingdom
}

\begin{abstract}
Spatiotemporal patterning of neural activity is thought to influence the development of connections in the visual pathway. This patterning can arise spontaneously or through sensory experience. Here, we use a combination of natural and simple stimuli to investigate which elements of the visual environment modulate the earliest responses in the primary visual pathway of developing ferrets. Recordings were made during the first 2 weeks of visual responsiveness, which, in the ferret, overlaps with the period that the eyelids have not yet opened. Even when the eyelids are closed, both thalamic and cortical activity was found to be temporally modulated under conditions of natural visual stimulation. The modulations correlated with temporal changes in stimulus contrast but also reflected spatial structure in the visual scene. Simple stimuli were used to show that early responses to naturalistic stimuli are influenced by the localization and structure of through-the-eyelid receptive fields. The early visual responses were also characterized by substantial variability in the ability of the cells to detect stimuli of different duration and different intensity, in a temporally precise manner. These temporal and spatial properties should constrain how plasticity mechanisms interpret naturally patterned activity.
\end{abstract}

Key words: visual development; natural scenes; dLGN; response properties; ferret; closed eyelids

\section{Introduction}

It is well established that early visual experience is important for the development of the primary visual pathway (Katz and Shatz, 1996). Furthermore, manipulation experiments in vivo indicate that the spatiotemporal properties of neural activity can instruct the refinement of connections in the visual system (Hubel and Wiesel, 1965; Sengpiel et al., 1999; Engert et al., 2002; Ruthazer et al., 2003). For instance, artificially induced strabismus, which effectively decorrelates the inputs from the two eyes, disrupts the cortical connections that subserve binocularity (Hubel and Wiesel, 1965), and artificially correlating all neural activity in the optic nerves of ferrets in the period immediately before, and after, eye opening leads to abnormal development of orientation selectivity in visual cortex (Weliky and Katz, 1997). Other features of the visual system seem to develop normally when sources of activity are removed (Crowley and Katz, 1999) or when spatiotemporal patterns within the neural activity are disrupted (Huberman et al., 2003). However, the evidence that patterned neural activity can be used in an instructive way to refine connections has become central to models of neural development (Stent, 1971; Miller, 1994; Shouval et al., 1997).

Inherent in these developmental models is the notion that the

\footnotetext{
Received April 4, 2003; revised 0ct. 14, 2003; accepted 0ct. 29, 2003.

This work was supported by grants from the Wellcome Trust. C.J.A. and M.S.G. were graduate students in the Wellcome Trust Programme in Neuroscience. We thank Darragh Smyth for programming the stimulus presentation software and for help with data analysis and Pat Cordery for expert technical assistance with histological procedures. We also thank David Tolhurst, Gary Baker, Kristine Krug, and members of the Thompson laboratory for discussions. Correspondence should be addressed to Dr. lan D. Thompson, University Laboratory of Physiology, Oxford University, 0xford 0X1 3PT, UK. E-mail: ian.thompson@physiology.ox.ac.uk.

C. J. Akerman's present address: Cold Spring Harbor Laboratory, Cold Spring Harbor, NY 11724

DOI:10.1523/JNEUROSCI.1002-03.2004

Copyright $\odot 2004$ Society for Neuroscience $\quad$ 0270-6474/04/240170-13\$15.00/0
}

"fidelity" of the spatiotemporal patterned activity dictates which neurons are coactive and that this constrains the level of specificity that can be attained. However, although the quantitative properties of spontaneous activity in the developing retina (Meister et al., 1991; Wong and Oakley, 1996; Feller et al., 1997; Lee et al., 2002), dLGN (Weliky and Katz, 1999), and visual cortex (Chiu and Weliky, 2001, 2002) have been well described, very little is known about the spatiotemporal properties of stimulusdependent patterned activity, particularly under naturalistic conditions. Here, we describe the response properties of single visual neurons during early development and ask how these properties might shape spatial and temporal activity patterns in the primary visual pathway. We have focused on the earliest visual responses during development, which, in the case of the ferret, begin before eye opening (Chapman and Stryker, 1993; Krug et al., 2001; Akerman et al., 2002). This period is particularly interesting because it has been shown that early visual experience, during the period before eye opening and immediately after eye opening, is important with respect to the development of the retinogeniculate pathway (Akerman et al., 2002) and the development of visual cortex (White et al., 2001).

Various neuronal properties will constrain the response to natural visual stimulation. Here, we investigate the temporal response properties and contrast sensitivities of developing neurons. We also investigate whether the response of a developing neuron is patterned by the spatial features of natural stimuli, an issue that is particularly relevant before eye opening, when the eyelids cause optical blurring. To address each of these questions, a combination of naturalistic and simple visual stimuli were used. The natural visual stimuli provide information about how the cells respond under (close to) normal conditions. Meanwhile, the simple stimuli allow us to systematically vary individual stimulus 
parameters and, thus, assess how each of these might contribute to the responses of the cells during natural vision in the developing visual system.

\section{Materials and Methods}

Animals, surgery, and anesthesia. Pigmented ferret kits were studied between postnatal day (P) 18 and P36; the day of birth was designated as P0. All animals were housed under a $16 / 8 \mathrm{hr}$ light/dark cycle. In vivo electrophysiological recordings were performed as described previously (Krug et al., 2001; Akerman et al., 2002). Briefly, anesthesia was induced by intramuscular injection of $2 \mathrm{ml} / \mathrm{kg} 0.9 \%$ alphaxalone and $0.3 \%$ alphadolone acetate (Saffan; Pitman-Moore, Uxbridge, UK). After cannulation of the radial vein, anesthesia was maintained by intermittent administration of Saffan (1:2 in $0.9 \%$ saline, i.v.), a tracheotomy was performed, and a tube was inserted directly into the trachea. For dLGN recordings, craniotomies (measuring $\sim 9 \mathrm{~mm}^{2}$ ) were centered $8-10 \mathrm{~mm}$ rostral and 4-6 mm lateral of lambda. For cortical recordings, craniotomies (measuring $\sim 2 \mathrm{~mm}^{2}$ ) were made $7-10 \mathrm{~mm}$ lateral and $0-2 \mathrm{~mm}$ rostral of lamda. During recording, anesthesia was maintained by continuous infusion of medetomidine (Domitor; 11-44 mg/kg/hr; Pfizer, Sandwich, UK) and ketamine (2.5-10 mg/kg/hr; Fort Dodge, Southampton, UK). Muscular paralysis was induced and maintained with gallamine triethiodide (Flaxedil; $10 \mathrm{mg} / \mathrm{kg} / \mathrm{hr}$; Sigma, Poole, UK) and delivered with the anesthetic in an infusion mixture that also contained $4 \%$ glucose and $0.18 \%$ sodium chloride (Aqupharm; $1.5 \mathrm{ml} / \mathrm{hr}$; Animalcare, York, UK).

Recordings. Action potentials were recorded extracellularly from isolated neurons with glass-coated tungsten microelectrodes with a $5 \mu \mathrm{m}$ exposed tip. Electrode penetrations in the dLGN were made vertically, and those in the visual cortex were made radial to the cortical surface at $\sim 16^{\circ}$ to the vertical. Flashes of light from an opthalmoscope (maximum luminance $\sim 500 \mathrm{cdm}^{-2}$ ) were used to identify visually responsive neurons and to classify cells as responding more to an increase in luminance ("On-dominated") or a decrease in luminance ("Off-dominated"). For all experiments, stimuli were presented to both eyes. We used hand-held stimuli to test for direct excitatory drive from both eyes but found no convincing evidence for this at any of the ages tested.

For quantitative studies, visual stimuli were presented on a Sony (or Eizo) computer display monitor controlled by a graphics card (Cambridge Research Systems, Rochester, UK). The screen had a presentation area of $396 \times 297(316 \times 237) \mathrm{mm}$ such that at the viewing distance of $28.5 \mathrm{~cm}$ the screen covered a large proportion of the putative visual field of the ferret $\left[69.6 \times 55.0^{\circ}\left(58.0 \times 45.2^{\circ}\right)\right]$. The stimulus display comprised $800 \times 600$ pixels such that each pixel subtended $\sim 0.1^{\circ}\left(0.08^{\circ}\right)$. From light meter readings, the minimum luminance of the screen was measured as $0(0) \mathrm{cdm}^{-2}$, and the maximum was measured as 108.4 $(72.6) \mathrm{cdm}^{-2}$. In practice, the reflectance of the screen would mean that the minimum luminance was not quite zero. Nevertheless, using these measurements, the maximum contrast that could be generated was defined as $100 \%$ Michelson contrast: Michelson contrast $=\left(L_{\max }-L_{\min }\right) /$ $\left(L_{\max }+L_{\min }\right)$, where $L$ is the luminance of a point on the display at a particular time. All statistical tests were two-tailed comparisons, and population values are reported as means \pm SEM or medians. Population data were tested for normality, and the appropriate nonparametric or parametric statistics were used.

At least two electrolytic lesions (3-4 $\mu \mathrm{A}$ for 3-4 sec) were delivered routinely at different points along electrode penetrations. At the end of an experiment, animals received an intravenous terminal dose of pentobarbitone sodium (Sagatal; RMB Animal Health, Harlow, UK) and were perfused transcardially with $4 \%$ paraformaldehyde. After cryoprotection, $50 \mu \mathrm{m}$ freezing microtome brain sections were cut and stained with cresyl violet. Electrolytic lesions were drawn under camera lucida and used to reconstruct electrode penetrations.

Cortical data were obtained from a total of 47 neurons. Thalamic data were obtained from a total of 180 neurons, 123 of which could be assigned histologically to geniculate laminae. Of these, 96 (78\%) were from the A layers and 27 (22\%) were from the C layers of the dLGN. Interestingly, in experiments in which we have a sufficient number of cells from both $\mathrm{A}$ and $\mathrm{C}$ laminae, there was no obvious difference in responses. To measure transmission properties of the closed eyelid, a portion of facial skin including the intact closed eyelid was removed from the animal during terminal perfusion and placed over a light meter with the eyelid positioned centrally. Attenuation by the closed eyelid was calculated as the luminance detected without the eyelid versus the luminance detected with the eyelid covering the light meter.

Naturalistic visual stimuli. Naturalistic stimuli designed to mimic typical neonatal ferret head movements were constructed as described previously (Akerman et al., 2002). Briefly, movies were constructed from sequences of digital photographs taken inside the animals' cages, and the temporal parameters of the movies were based on video footage of awake and behaving ferret kits. Each digital photograph was scaled such that its maximum luminance did not exceed the reflected luminance of the home cage $\left(48 \mathrm{~cd} \mathrm{~m}^{-2}\right)$, and each image represented a rotation of $10^{\circ}$ from the previous image. The first and last of these were presented for $2200 \mathrm{msec}$ to simulate the animal's head being still, and each of the central nine images was presented for $110 \mathrm{msec}$ (i.e., one frame length) such that the entire movie simulated a head movement through $100^{\circ}$ of visual space over a total of $1100 \mathrm{msec}$. For recording experiments, the movie was presented as part of a sequence in which the first half consisted of a blank screen at the mean luminance of the movie ("blank"). Three different movies were constructed, and responses were collected from $40-100$ repetitions of a movie.

To test the spatial sensitivity of responses, a series of "unstructured" movies were used. Unstructured movies had identical temporal properties to the "structured" movies described above, but they differed in their spatial properties: whereas each frame of the structured movies consisted of a digital photograph, each frame of the unstructured movie was blank, with a luminance equal to the mean luminance of the corresponding structured movie frame. For these experiments, the movie types were interleaved such that each structured movie sequence was followed by the matched unstructured movie sequence. Responses were compared over at least 20 repeats of both movie types, and a neuron was classed as "structure sensitive" if it discharged a significantly different number of spikes across the two movies ( $p<0.05$; Mann-Whitney $U$ test) or responded differently to one of more of the corresponding frames $(p<$ 0.05; Dunn post test after Kruskal-Wallis nonparametric ANOVA). To compare cells, an index of structure sensitivity was calculated as:

$$
\text { Structure sensitivity }=\sum_{\mathrm{i}} \frac{\left|s_{\mathrm{i}}-u_{\mathrm{i}}\right|}{\sum s+\sum u},
$$

where $s$ is response to the structured movie, $u$ is response to the unstructured movie, and $i$ is the movie frame bin.

Whole-field flashed stimuli. To investigate temporal summation, latency, and precision of responses, single whole-field flashed stimuli were used in which the time that the stimulus was presented (the stimulus duration) was varied systematically. The time the background was presented between stimuli was kept constant $(4000 \mathrm{msec})$. The stimuli were presented in the optimal polarity for each cell, either as bright flashes for On-dominated cells (bright whole-field flashes on a dark background) or as dark flashes for Off-dominated cells (dark whole-field flashes on a bright background). Latency and precision were calculated from responses to long flashes $(500-1500 \mathrm{msec})$. To investigate stimulus duration, between 7 and 15 different flash lengths (from 10 to $1500 \mathrm{msec}$ ) were presented to each cell. The order in which the stimuli were presented was randomized, and each stimulus was presented at least 10 times. To study responses to different temporal contrasts, flashed wholescreen stimuli were presented about a constant mean luminance. Ten different temporal contrasts between 0 and 100\% (Michelson contrast; see above) were presented in a randomized order. For each contrast, the screen flashed from dark to bright every $2 \mathrm{sec}$ (a temporal frequency of $0.25 \mathrm{~Hz}$ ), and responses were recorded for a total of 20 cycles of each stimulus. A uniform display of $0 \%$ contrast and mean luminance was used to record spontaneous activity.

Receptive field mapping. Receptive field (RF) structure was investigated using reverse correlation techniques (Jones and Palmer, 1987). Randomized sequences of black (Off) and white (On) flashed squares $\left(29 \times 29^{\circ}\right)$ were presented for 500 or $1000 \mathrm{msec}$ at 48 positions on a blank gray 
background. With the screen size used, the stimuli overlapped and, thus, enabled RFs to be described with a resolution of $9.9 \times 9.9^{\circ}$ of visual space (i.e., the size of each pixel in the RF profiles). All responses recorded during the stimulus presentation time were included in the analysis, and responses over at least 10 repeats of the stimulus set were analyzed for each cell. Spikes recorded during a $10 \mathrm{sec}$ blank screen period were used as a measure of spontaneous activity. Response-weighted spatial profiles for both On and Off stimuli were generated. The response at all pixel positions was normalized by dividing by the total number of responses for the strongest stimulus, whatever the position or polarity of that stimulus. The Off profile for each cell was subtracted from its On counterpart to produce a combined RF profile. The combined RF was then smoothed with a Gaussian filter, chosen such that within the distance between two adjacent pixels of the original profile, the Gaussian decayed to $<10 \%$. RFs at this early stage of development cover a large part of visual space $\left(30-70^{\circ}\right)$. Despite using large display monitors, it was still necessary to adjust the position of the monitor for each cell to record as much of each $\mathrm{RF}$ as possible. This meant that the monitor was not in a constant location relative to the animal, and, as a result, the RF locations do not reflect absolute positions in visual space. The open eyelid population data at P32-34 has been published previously (Akerman et al., 2002).

We applied four measures to RFs. First, "RF smoothness" was the correlation coefficient across all unique combinations of neighboring pixels in the RF profile, excluding pixel positions with zero stimulus sensitivity. RF smoothness values vary between -1 and +1 , with positive values indicating RFs that had spatially continuous regions of the same response polarity. Random RFs were generated by scrambling the pixels of real RFs. Second, to estimate "RF radius," the unsmoothed dominant polarity map was fit by a least squares minimalization algorithm using a two-dimensional Gaussian curve of the form:

$$
W(x, y)=a \cdot e^{-\left(x / r_{x}\right)^{2}} \cdot e^{-\left(y / r_{y}\right)^{2}},
$$

where $W$ is a weighting function describing the dominant polarity map center, $x$ and $y$ are positions in the RF, $a$ is amplitude, and $r_{\mathrm{x}}$ and $r_{\mathrm{y}}$ are the radii of the function where it falls to $1 /$ e of its maximum in the $x$ and $y$ directions, respectively (Grubb and Thompson, 2003). For cells in which the Gaussian fit was judged to be satisfactory, RF radius was estimated as the square root of the product of $r_{\mathrm{x}}$ and $r_{\mathrm{y}}$. Third, the "On-Off ratio" captured the relative strength of each response polarity and was defined as:

$$
\text { On-Off ratio }=\frac{\left|\left(O N_{\text {total }}\right)-\left(O F F_{\text {total }}\right)\right|}{\left|\left(O N_{\text {total }}+O F F_{\text {total }}\right)\right|},
$$

where $O N_{\text {total }}$ and $O F F_{\text {total }}$ denote the summed response to all white stimuli and all black stimuli, respectively (Akerman et al., 2002). Fourth, a measure of On-Off separation was used to quantify the spatial segregation of the On and Off subfields:

$$
\text { On-Off separation }=1-\frac{\left|\sum_{\mathrm{i}}\left(O N_{\mathrm{i}}^{*} O F F_{\mathrm{i}}\right)\right|}{\|O N\| \star\|O F F\|}
$$

where $O N_{\mathrm{i}}$ and $O F F_{\mathrm{i}}$ denote the sensitivity to bright and dark stimuli, respectively, at spatial position i of the RF map, and:

$$
\|O N\|=\sqrt{\sum_{\mathrm{i}} O N_{\mathrm{i}}^{2}}
$$

denotes the norm magnitude of the vector of ON spatial sensitivities. More specifically, On-Off separation is one less the normalized dot product of the On and Off sensitivity maps. It is maximized when the maps are segregated to different spatial locations and minimized when the maps occupy the same positions (Akerman et al., 2002).

\section{Results}

Recordings were made during the first 2 weeks of visual responsiveness, which, in the ferret, overlaps with the period when the eyelids are still closed. The first visual responses can be recorded at P19, and the eyelids open at approximately P32 (Krug et al.,
2001, Akerman et al., 2002). Because the response characteristics under normal conditions were the central focus for this study, the eyelids were left in their natural state for the recordings (i.e., the eyelids were left closed if the animal's eyes had not yet opened and were left open if, by the time of the experiment, the eyelids had opened), except where indicated.

\section{Natural stimuli pattern early visual responses in thalamus and cortex}

Natural movie stimuli were constructed from sequences of digital images from inside the animals' home cage environment, and the temporal parameters of the movies were based on measurements of typical ferret kit head movements (Fig. 1A) (see Materials and Methods). These stimuli were then presented to anesthetized ferret kits (age, P19-32), while extracellular single-unit recordings were made in either the dLGN or primary cortex. For analysis purposes, each movie was divided into two separate periods (Fig. 1, gray): a baseline "spontaneous period" recorded during the presentation of a blank screen and a "movie period" that encompassed the simulated head movement. In the raster plots shown in Figure 1, the time-locked spikes between 0 and $1 \mathrm{sec}$ are responses to the onset of the blank screen, and time-locked spikes between 6 and $7 \mathrm{sec}$ are responses to the onset of the first frame of the movie. These spikes did not fall within either the spontaneous or the movie periods and, therefore, were not included in the analyses.

As has been shown previously (Akerman et al., 2002), neurons in the geniculocortical pathway were found to respond robustly to movies designed to mimic neonatal ferret head movements, even when presented through the naturally closed lids. Figure $1, B$ and $C$, show example recordings from the dLGN and visual cortex, respectively. The first two example cells from the dLGN show that the average firing rate was elevated under natural visual stimulation compared with spontaneous activity. Across all dLGN cells and all movies, the median firing rate during the movie period was 0.66 spikes per second, whereas the median firing rate during the spontaneous period was 0.14 spikes per second $(n=$ 90 neurons). Of course, the average firing rate captures only one aspect of visual responsiveness. For instance, the third dLGN cell in Figure $1 B$ has high spontaneous activity, and the mean level during the movie is only slightly elevated. Importantly, however, the response pattern of the cell during the movie is strongly temporally modulated.

This response modulation to the movie stimuli, which can be seen in all three examples in Figure $1 B$, was a common feature of geniculate responses. To capture the temporally locked responses, we binned the movie period by frame length (110 msec) and analyzed whether spike rate showed statistically significant modulation over time ( $p<0.05$; Kruskal-Wallis nonparametric ANOVA). Sixty-seven of 90 dLGN neurons (74\%) showed statistically significant modulation in their spike rate during the movie period (only $6 \%$ of neurons showed variation across time bins during the spontaneous period). This means that the responses of dLGN neurons were temporally locked to the natural stimulation parameters.

Cortical recordings during a similar period (age, P24-31) demonstrated that, as in the dLGN, cells in the visual cortex responded reliably at specific phases of the movie period. The three neurons in Figure $1 C$ are typical of the responses in cortex in that they showed relatively low levels of spontaneous activity but responded in a stimulus-locked manner during the movie period. Of the 47 cortical neurons recorded, 36 (77\%) exhibited statistically significant modulation in their spike rate during the 

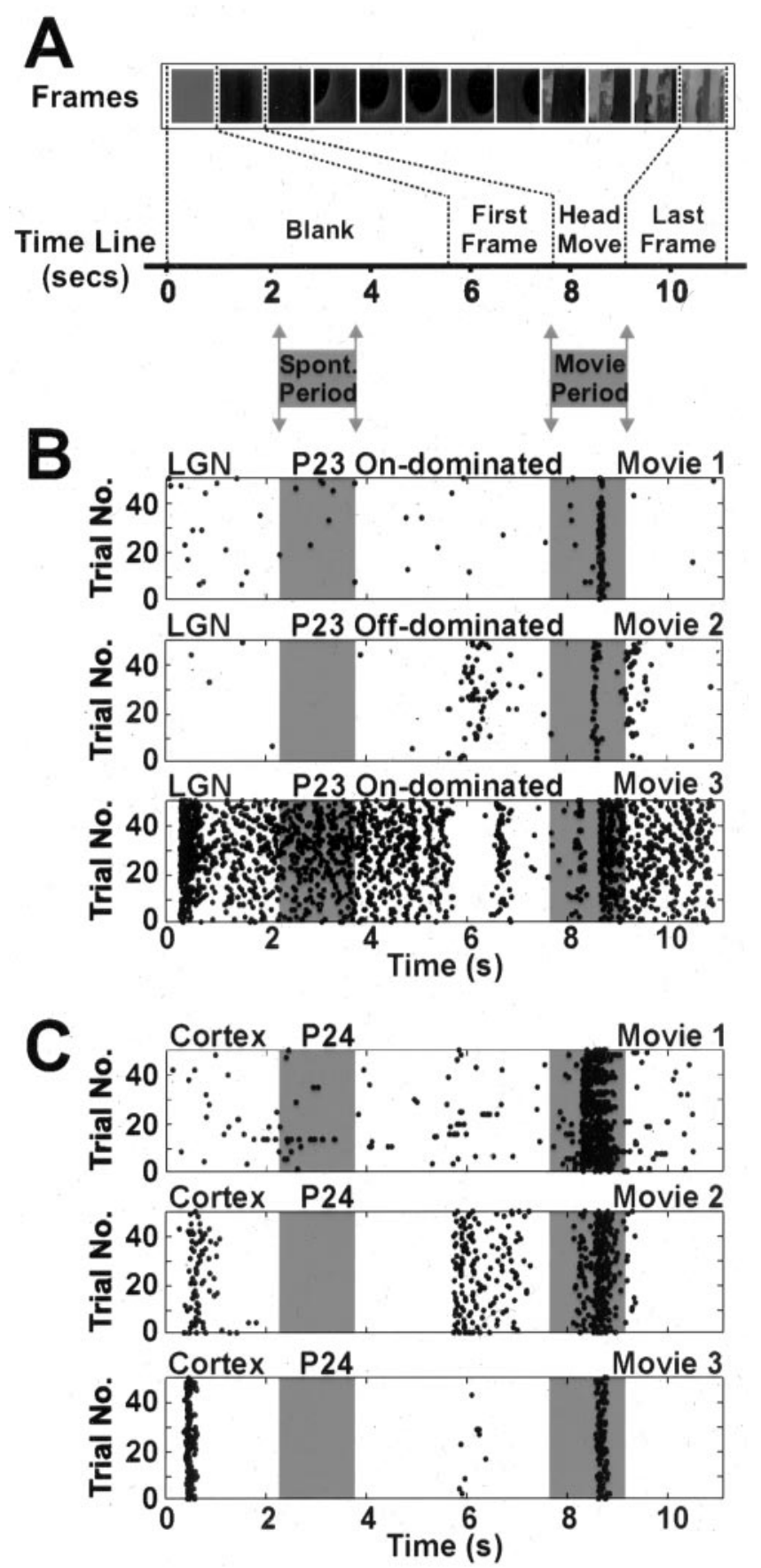

Figure 1. $\quad \mathrm{dLGN}$ and cortical responses to natural movies through closed eyelids. $A$, Short movies were constructed that mimicked the spatiotemporal visual stimulation resulting from a typical neonatal ferret head movement. Temporal parameters for the movies were based on video footage of ferret kits (P18 -24) behaving in their home cage environment (see Materials and Methods). In each movie, this behavior was simulated with a sequence of digital images from the home cage, each image representing a rotation of $10^{\circ}$. To record spontaneous activity, a blank screen at the mean luminance of the digital images (blank) was presented for $5.5 \mathrm{sec}$ at the start of each movie sequence. For analysis, each movie sequence was divided into two parts, the spontaneous period and the movie period. The movie period encompassed the simulated head movement and ended four frame lengths (i.e., $0.44 \mathrm{sec}$ ) into the final frame. The spontaneous period matched the movie period in length but was taken from the middle of the blank. $B$, Examples of dLGN responses when movie stimuli were presented to the closed eyelids. Raster plots are shown for three different dLGN cells recorded $9 \mathrm{~d}$ before the eyes open. Each dot represents a single spike. All of the cells show modulated responses during the movie period but not during the spontaneous period (indicated by shaded regions). Stated at the top of each raster are the age, the cell type, and the movie code. C, Movie responses through closed eyelids for three different cortical cells recorded at P24. As in the dLGN, cortical cells respond strongly movie period $(p<0.05$; Kruskal-Wallis nonparametric ANOVA). No cortical neuron showed variation across time bins during the spontaneous period. Across all cortical cells and all movies, the median firing rate during the movie period was 0.18 spikes per second, whereas the median firing rate during the spontaneous period was 0 spikes per second.

The results from the movies demonstrate that even at this early stage, the system is sensitive to changes in the visual scene and is able to relay this information with sufficient fidelity to drive geniculate and cortical cells in a stimulus-locked manner. However, from these data it is not obvious how properties of the developing visual system shape the response patterns. Below, we used simple stimuli to explore the temporal, contrast, and spatial response properties that could constrain the neuronal responses to natural stimuli. All subsequent experiments focused on the dLGN.

\section{Temporal properties of early visual responses}

Temporal response properties of neurons in the developing visual system will influence the probability that their activity patterns will be correlated during periods of sensory experience. For instance, variability in the response times across geniculate cells will affect whether they are able to activate a common postsynaptic cortical cell at the same time. Also, differences in stimulus sensitivity, or in the response duration to a particular stimulus event, could change the probability that two neurons are coactive. Here, we have investigated how these parameters vary in developing dLGN neurons.

\section{Response latency and precision}

To quantify the latency and temporal precision of early dLGN responses, flashed whole-field stimuli were presented to ferret kits, and the times of the first action potentials fired to these stimuli were analyzed (Fig. 2A). Stimulus polarity (On or Off) was always optimal for the cell (see Materials and Methods). We concentrated on the timing of first spikes for two reasons. First, comparing the times of single spikes is simpler than comparing bursts of spikes. Second, in terms of synaptic modification by natural spike trains, the timing of the first spike may be the most important (Froemke and Dan, 2002). The times of first spikes were presented as raster plots and, in an attempt to extract measures that were resistant to variability in failure rates and levels of spontaneous activity, a first spike histogram was constructed (Fig. 2A, bottom) (Guido and Sherman, 1998; Jenison et al., 1998). Many cells displayed a single response such that there was one stimulus-locked, vertical cluster of events on the first spike raster plot (with some variability in spike time across trials) and a corresponding strong peak in the first spike histogram (see the first cell in Fig. 2 A). In other cells, as well as the strong peak in the first spike histogram, there was evidence of a second smaller peak (see the second and third cells in Fig. 2A). The spikes in the secondary peaks may reflect the latencies of different retinal ganglion cell (RGC) inputs to the same dLGN cell, or could reflect the responses of the cell in different response modes (i.e., tonic vs burst) (Guido and Sherman, 1998).

The "latency" of the dLGN cells was defined as the time of the peak of the histogram. The "precision" was defined as the mini-

$\leftarrow$

during the movie period but not the spontaneous period. Phase-locked spikes between 0 and 1 sec of the movie rasters are responses to the onset of the blank screen. Phase-locked spikes between 6 and $7 \mathrm{sec}$ are responses to the onset of the first frame of the movie. None of these spikes were part of the spontaneous period or movie period and, therefore, were not included in any analyses. 
mum width (in milliseconds) of a window about the latency of the cell that captured $50 \%$ of the first spikes. Figure $2 B$ plots the distribution of precision and latency values for dLGN neurons $(n=75)$ recorded during the first 2 weeks of visual responsivity (P19-34). During this early period of development, response latencies of dLGN cells tended to be long and showed considerable variation across neurons: the median latency was $268 \mathrm{msec}$, and the interquartile range was $271 \mathrm{msec}$ (minimum, 36; maximum, 891). For comparison, a typical response latency in adult $\mathrm{dLGN}$ is $\sim 35 \mathrm{msec}$ (our unpublished observations). Similarly, precision values were high and varied across the developing cells. Between P19 and P34, the median cell fired $50 \%$ of its first spikes over a window of $54 \mathrm{msec}$ (minimum, 2.3; maximum, 424), and the interquartile range for the population was $91 \mathrm{msec}$. The fact that the majority of the cells (72\%) showed precision values of $\leq 100 \mathrm{msec}$ is consistent with the temporally modulated responses observed under natural visual stimulation (Fig. 1). However, the values of latency and precision indicate a wide range of response times under visual stimulation, which has implications for timing-based mechanisms of developmental plasticity (see Discussion).

The scatter plots in Figure 2, $C$ and $D$, show that within the relatively narrow time window investigated here, there were significant developmental trends in both latency and precision: younger cells have longer response latencies, and their responses are less temporally precise than dLGN neurons from older animals (latency Spearman $r=-0.55 ; p<0.0001$; precision Spearman $r=-0.24 ; p<0.05)$. Indeed, over the 2 weeks between approximately P20 and P34, the median response latency more than halved from 308 to $136 \mathrm{msec}$, and the median precision fell from 43 to $16 \mathrm{msec}$. The developmental trend toward decreasing response latencies was also evident when the analysis was restricted to cells recorded through the naturally closed eyelids (latency Spearman $r=-0.38 ; p<0.005$ ). One possible explanation for such a developmental trend before eye opening is that changes in the optical properties of the eyelids allow increasing amounts of light to be transmitted in older animals. However, measurements of the transmission properties of the closed eyelids (see Materials and Methods; $n=12$ ) actually revealed a negative correlation between age and transmission (Spearman $r=-0.56 ; p=0.06$ ). The fact that older eyelids tend to transmit less, not more, light than younger eyelids suggests that the changes underlying the developmental trends are neural in origin.

Summation threshold and response duration

To further characterize the temporal properties of early visual responses, we tested how well the system can detect single flashes of different duration. We also asked how sustained the responses
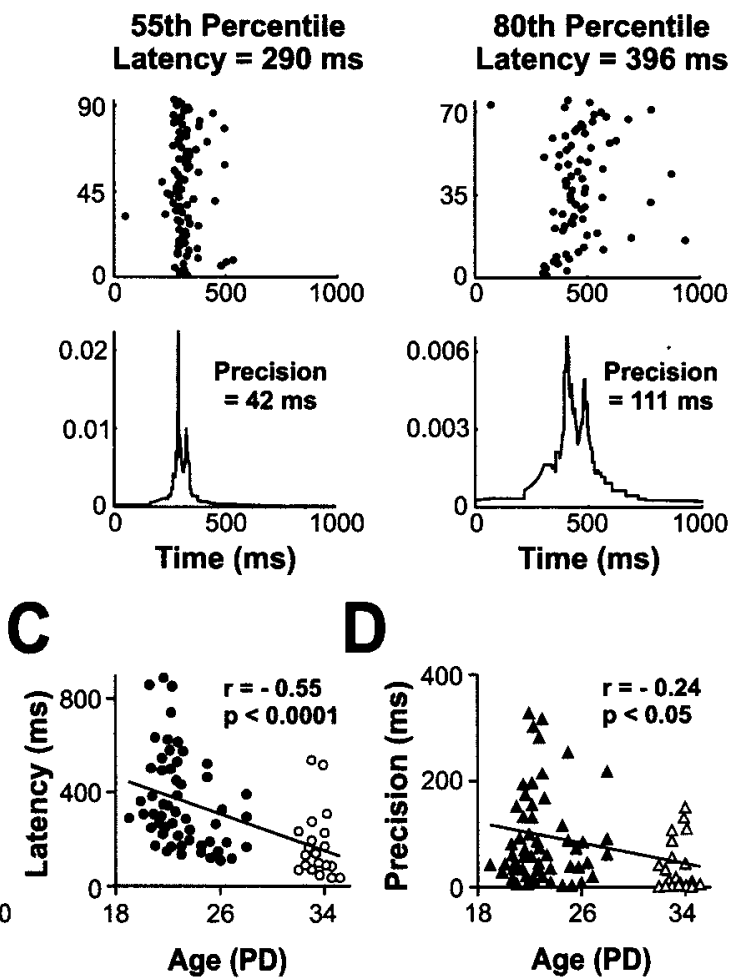

Figure 2. Latency and precision of the earliest visual responses. Latency and precision of dLGN cells were calculated from the first spike times in response to whole-field flashed stimuli. The optimal stimulus polarity of the cell was used (bright flashes for $0 \mathrm{n}$ dominated or dark flashes for Off-dominated). A, dLGN cells were ranked by latency, and for the cells at the 20th, 55th, and 80th units and calculated as the inverse of the width of the window required to capture 10 spikes) that a first spike was recorded at a particular time after the onset of the flash. The latency was defined as the time of the peak of the histogram, the precision as the midth of a window about the latency that captured half of the first spikes. $B$, Cumulative probability plot of latency and atencies (minimum, 36 msec; median, 268 msec; maximum, $891 \mathrm{msec}$ ) and precision values (minimum, $2.3 \mathrm{msec}$; median, 53 ( Latency and precision showed a statistically significant trend over this developmental period with both measures decreasing with age (latency Spearman $r=-0.55 ; p<0.0001$; precision Spearman $r=-0.24 ; p<0.05$ ). For presentation purposes, overlapping data points in the scatter plots were offset along the $x$-axis.

of cells were to stimuli. In these experiments, the time that the stimulus was presented (the "stimulus duration") was systematically varied, whereas the interstimulus period was constant and long ( $4 \mathrm{sec}$ ). Between 7 and 15 different stimulus durations ranging from 10 to $1500 \mathrm{msec}$ were presented to each cell, and the "summation threshold" of a cell was defined as the shortest stimulus duration to evoke a significant increase in spike number compared with spontaneous activity ( $p<0.05$, Mann-Whitney $U$ test). The "response duration" of a cell was based on the response to the $1000 \mathrm{msec}$ flash and was defined as the period of time during which the activity of a cell remained above spontaneous levels. Figure $3 A$ shows dLGN cell responses to flashed stimuli of different duration recorded before eye opening. Raster plots were constructed by combining the responses from 10 presentations of each stimulus duration, and the summation threshold is stated above each raster. Note the differences in scales on the $y$-axes of the raster plots. The cell at the 70th percentile includes responses to a $1000 \mathrm{msec}$ flash and illustrates how sustained the responses could be (the response duration of this cell was $739 \mathrm{msec})$. 
A

\section{0th Percentile Threshold $=20 \mathrm{~ms}$}

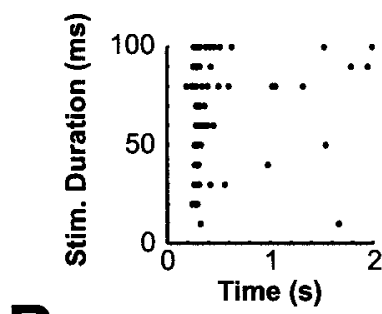

B
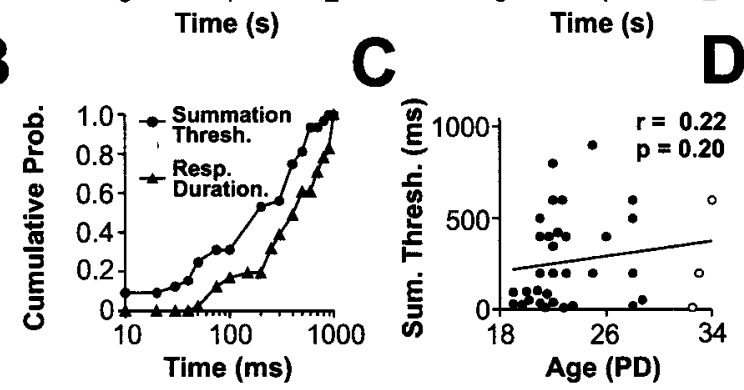

Figure 3. Temporal properties of the earliest visual responses. dLGN cells were presented with whole-field flashed stimuli of different duration, and the summation threshold of a cell was defined as the shortest stimulus to evoke a statistically significant increase in spike rate compared with spontaneous activity ( $p<0.05$; Mann-Whitney $U$ test). The response duration of a cell was calculated from responses to a 1000 msec stimulus and was defined as the period of time during which the activity of the cell remained above spontaneous levels. $A, \mathrm{dLGN}$ cells were ranked according to their summation threshold, and raster plots of the cells at the 10th, 35th, and 70th percentiles are shown. Each raster plot was constructed by combining the responses recorded from 10 presentations of each stimulus duration. Stated above each raster is the summation threshold for that cell. Note different scales on the $y$-axes. $B$, Cumulative probability plot of the summation threshold and response duration of dLGN cells recorded during the first 2 weeks of visual responsiveness. $C, D$, Scatter plots comparing age against the summation threshold $(C)$ and response duration $(D)$. Summation threshold did not correlate with age over this developmental period (Spearman $r=0.22 ; p=$ 0.20 ), whereas response duration correlated significantly with age (Spearman $r=0.50 ; p<0.005$ ). Other conventions are as shown in Figure 2.

Figure $3 B$ plots the distribution of all summation thresholds and response durations for dLGN neurons recorded during the first 2 weeks of visual responsiveness $(n=35$ and 36, respectively). Considering summation threshold first, the cell at the 50th percentile responded to stimulus durations of $\geq 200 \mathrm{msec}$. This suggests that a single frame of the movie stimuli $(110 \mathrm{msec}$ duration) (Fig. 1) would not be sufficient to alter the activity of the majority of developing dLGN neurons; instead, the cells appear to integrate over two or more movie frames to produce a response. However, dLGN cells before eye opening did vary greatly in their temporal sensitivity. Some neurons were able to respond to extremely short stimuli. The neuron at the 10th percentile (Fig. 3A), for instance, could reliably detect flashes that were $20 \mathrm{msec}$ in duration. Figure $3 B$ also reveals the considerable variability in the response duration of cells to the $1000 \mathrm{msec}$ flash. The cell with the briefest response fired action potentials for only $50 \mathrm{msec}$. In contrast, some cells displayed sustained elevated firing for $>900 \mathrm{msec}$. Over the first 2 weeks of visual responsiveness, there was no robust correlation between age and summation threshold (Spearman $r=0.22 ; p=0.2$; Fig. 3C). The response duration showed a positive correlation between age and response duration (Spearman $r=0.50 ; p<0.002$ ) (Fig. 3D), such that older cells tended to exhibit longer spike trains to a 1000 msec stimulus than younger cells.

We next asked whether the different aspects of temporal responses described in Figures 2 and 3 showed any interdependence. As shown in Figure $4 A$, the latency of a cell was found to be strongly correlated with the temporal precision of its responses (Spearman $r=0.74 ; p<0.0001 ; n=75$ ). For instance, a dLGN neuron with a latency of $100 \mathrm{msec}$ would be expected to discharge half of its first spikes within a $20 \mathrm{msec}$ time window, whereas for a cell with a latency of $400 \mathrm{msec}$, the corresponding time window would be $\sim 100 \mathrm{msec}$. As shown in Figure $4 B$, the latency was also strongly correlated with the summation threshold (Spearman $r=0.45 ; p<0.01 ; n=35)$, indicating that cells that respond quickly are more sensitive to shorter stimulus events. No correlation was observed between the latency and the response duration (Fig. 4C) (Spearman $r=-0.28 ; p=0.10 ; n=36$ ). Taking these data together, the implication is that latency not only distinguishes cells based on when they respond after a stimulus event but also how precisely they respond and the range of stimuli that they can detect.

\section{Contrast sensitivity of early visual responses}

As well as temporal properties, the way developing cells behave over a range of stimulus intensities could be an important variable underlying responses to natural stimuli. We investigated the sensitivities of dLGN neurons to contrast using wholefield flashed stimuli in which the contrast was varied systematically (from 10 to 100\% Michelson contrast; see Materials and Methods). The term "whole-field contrast" is used to refer to this stimulus feature. The whole-field contrast threshold of a cell was defined as the lowest contrast at which the cell showed a statistically significant increase in firing compared with spontaneous activity ( $p<0.05$; Mann-Whitney $U$ test). Figure $5 A$ shows examples of the contrast stimuli and raster plots of corresponding responses from three dLGN neurons recorded during the first 2 weeks of visual responsiveness. From the examples, it can be seen that cells differed in terms of the strength and the sustained nature of their responses to different whole-field contrasts. The cell at the 20th percentile, for example, responded robustly across a wide range of stimuli, although with progressively fewer spikes as contrast decreased. Meanwhile the cell at the 80th percentile responded reliably to high contrasts but showed substantial failures at whole-field contrasts of $\leq 60 \%$. The population data for dLGN neurons between P19 and P34 is plotted in Figure $5 B$ and shows that over half of the cells had contrast thresholds of $\leq 30 \%$. As the scatter plot in Figure $5 C$ shows, over the developmental period studied here, there was not a strong correlation between age and contrast sensitivity (Spearman $r=$ $-0.22 ; p=0.16 ; n=43$ ).

These data show that even during the period of ferret development when the eyelids are still closed, neurons in the dLGN can respond to relatively small whole-field contrasts. Does this sensitivity to changes in whole-field illumination explain neuronal responses to natural movies presented to the closed eyelids? One approach is to examine the relationship between the neuronal response and the mean luminance of successive frames of the natural movies. Figure $6 \mathrm{~A}$ shows this relationship for one neuron recorded before eye opening (age, P26), in which the response of the cell (adjusted for the flash latency of the cell) is plotted against the difference in luminance between successive frames ("delta 

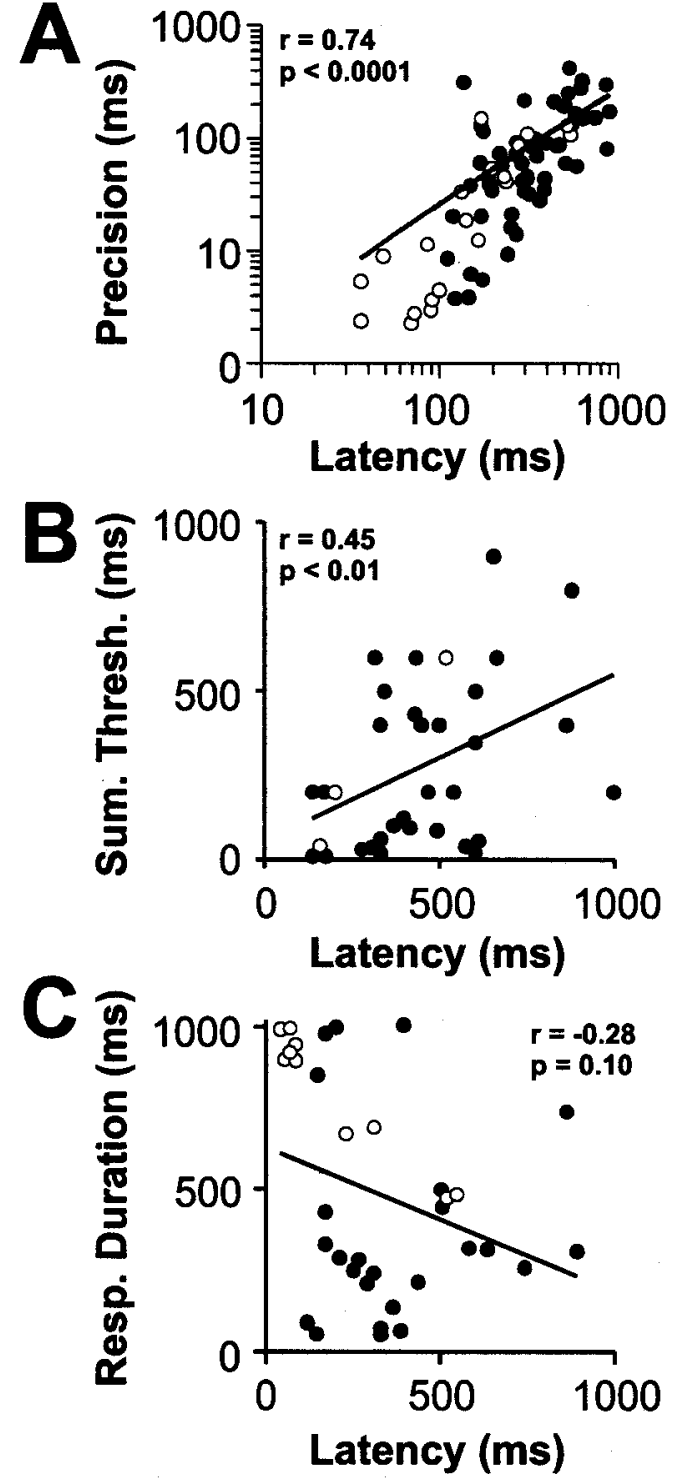

Figure 4. Response latency predicts other temporal response properties of a developing cell. Scatter plots of response latency versus precision $(A)$, summation threshold $(B)$, and response duration ( $C$ ) for dLGN cells recorded during the first 2 weeks of visual responsiveness. Cells recorded through the naturally closed eyelids are indicated with closed symbols, and those recorded just after eye opening are indicated with open symbols. The lines are unconstrained regression fits. The latency of a cell was found to be strongly positively correlated with both its precision (Spearman $r=0.74 ; p<0.0001 ; n=75$ ) and its summation threshold (Spearman $r=0.45 ; p<0.01 ; n=35)$. Latency and response duration were not significantly correlated (Spearman $r=-0.28 ; p=0.10 ; n=36$ ).

luminance"). In this example, delta luminance predicts both the increase in response over frames 8 and 9 , and the subsequent decrease in firing rate.

In Figure $6 B$, mean correlation coefficients between delta luminance and neuronal response are plotted separately for a population of On-dominated and Off-dominated cells. This data set was recorded before eye opening (P20-30) and was composed of cells in which both movie and latency data (as shown in Fig. 2) was collected. The mean correlation coefficient between Ondominated cell responses and delta luminance was positive (mean Spearman $r=0.36 \pm 0.06 ; n=13$ ) and significantly greater than zero ( $p<0.0005$; Wilcoxon), demonstrating that increased activity in On-dominated cells correlates with increases in whole-field luminance during the movies. Furthermore, the mean correlation coefficient for a smaller set of Off-dominated cells was negative (mean Spearman $r=-0.40 \pm 0.21 ; n=3$ ), suggesting that these cells were detecting decreases in luminance. This somewhat simplistic approach to the data does suggest that immature On and Off cells are acting as detectors of whole-field luminance change, but is this all that they are detecting?

\section{Early visual responses of single neurons encode spatial information}

In the preceding analyses, the change in whole-field luminance during the natural visual stimulation was found to be a good, although not perfect, predictor of the temporal pattern of dLGN responses. This raised the question as to whether dLGN neurons in developing ferrets before eye opening are merely detecting whole-field luminance changes through the closed eyelids, or whether these cells can actually detect spatial structure in natural scenes. To address this directly, we compared the responses to interleaved repeats of two types of movies that differed only in their spatial structure. Structured and unstructured movies shared identical mean luminance and temporal characteristics, but whereas frames of the structured movies contained spatial structure, each frame of the unstructured movie was blank, with a luminance equal to the mean luminance of the corresponding frame in the structured movie (see Materials and Methods). Figure $7 A$ shows an example of a structured and corresponding unstructured movie stimulus. Presentation of the two types of movie was interleaved, and spikes recorded during both types of movie were compared directly.

A neuron was classed as structure sensitive if it discharged a significantly different number of spikes across the entire structured movie ( $p<0.05$; Mann-Whitney $U$ test) or if it responded differently to one or more of the corresponding frames $(p<0.05$; Dunn post test after Kruskal-Wallis nonparametric ANOVA). The three example cells shown in Figure $7 B$ were all structure sensitive: cell 1 discharged more spikes across the structured movie than across the unstructured movie. Cell 2 did not show a difference in the total numbers of spikes, but it is clear from the raster plots that its peak response occurred at a different time during the two movies. Thus, when the raster plots were binned by frame length $(110 \mathrm{msec})$, this cell showed a statistically different response pattern to the structured and unstructured movies. Finally, both the overall firing rate and the temporal pattern of spikes for cell 3 were different during presentation of the two movies. Across a population of 44 movie-responsive dLGN neurons recorded before eye opening (P25-30), 28 (64\%) satisfied the statistical criteria for structure sensitivity.

To compare the degree to which the responses of different cells were modulated by spatial structure within the movie, we also developed an index of structure sensitivity (see Materials and Methods). This index measured the mean difference in responses to the structured and unstructured frames of the movies, returning higher values when the response to structured and unstructured movie frames was very different. For example, of the three cells in Figure $7 B$, cell 2 had the highest structure sensitivity value (0.87). This reflects the fact that the responses of cell 2 to the structured and unstructured movies were almost completely nonoverlapping in time. Figure $7 C$ plots the structure sensitivity index against age for the population of cells $(n=44)$ and shows that the cells most affected by spatial structure were recorded in the older animals, just before eye opening. However, there was only a weak positive correlation between structure sensitivity and age (Spearman $r=0.10 ; p=0.55$ ). 


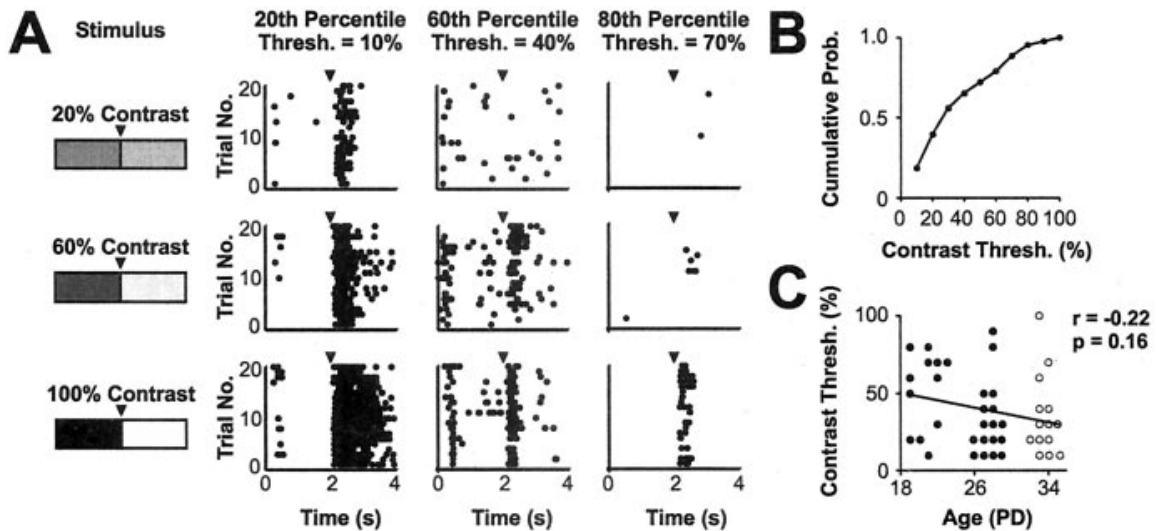

Figure 5. Contrast sensitivity of the earliest visual responses. Whole-field flashed stimuli were presented at a series of contrasts about a fixed mean luminance. The flashed contrast threshold was defined as the lowest contrast at which a neuron showed a significant increase in firing rate when compared with a blank screen at mean luminance ( $p<0.05$; Mann-Whitney $U$ test). Stimuli were presented at a temporal frequency of $0.25 \mathrm{~Hz}$. A, Neurons were ranked according to their contrast threshold, and raster plots for cells at the 20th, 60th, and 80th percentiles are presented (left to right) showing responses to 20,60, and 100\% contrasts (top to bottom). Each raster was constructed from a total of 20 cycles of the stimulus. $B$, Cumulative probability plot of the flashed contrast threshold of dLGN cells recorded during the first 2 weeks of visual responsiveness. The distribution reveals the range of thresholds displayed by cells during this period of development: the most sensitive cell responded to a contrast of just $10 \%$, and the least sensitive to a contrast of $90 \%$. C, Scatter plot showing that contrast threshold and age correlated negatively and weakly over this developmental period (Spearman $r=-0.22 ; p=0.16 ; n=43$ ). Other conventions are as shown in Figure 2 .
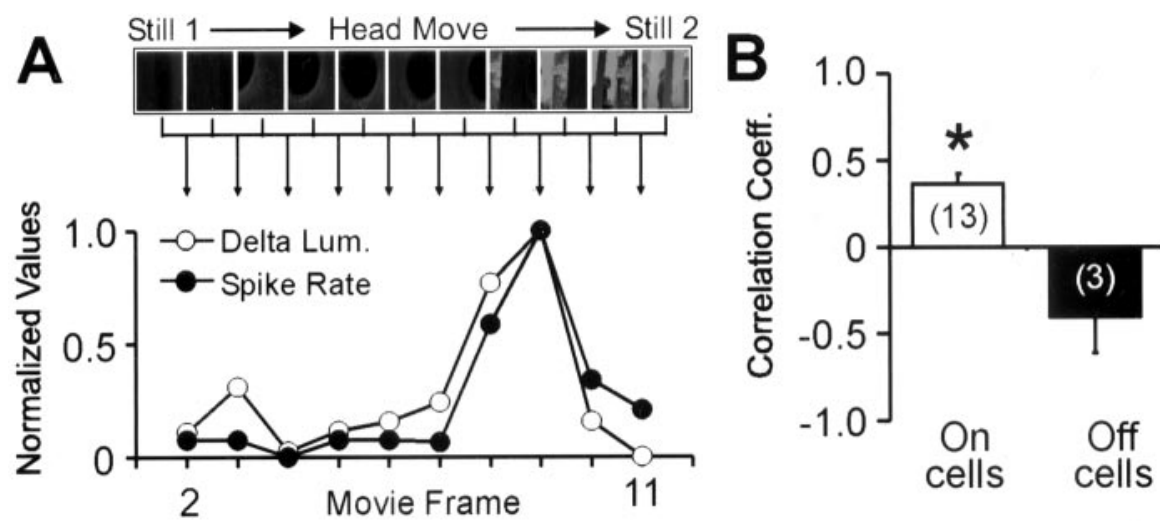

Figure 6. Correlating spike rate with luminance changes during a natural visual stimulus. $A$, We tested the correlation between the spike rate of a cell and delta luminance, the signed difference in mean luminance from two successive movie frames. The spikes of each cell were shifted backward by subtracting the latency (calculated as in Fig. 2), averaged over at least 50 repeats, and binned by movie frame length $(110 \mathrm{msec})$. The example plot shows the positive correlation between the spike rate recorded from an On-dominated P26 dLGN neuron and delta luminance during the corresponding movie (Spearman $r=0.49$ ). B, Delta luminance predicts a major component of responses to natural movie stimuli. Mean correlation coefficients between delta luminance and latency-shifted responses are plotted separately for $0 \mathrm{n}$ - and 0 ff-dominated cells. The responses of 0 n-dominated cells correlated strongly and positively, consistent with these cells responding more to increases in mean luminance. Meanwhile, the responses of Off-dominated cells correlated negatively, consistent with these cells responding more to decreases in mean luminance. The asterisk denotes that the 0 -dominated mean is significantly different from zero ( $p<0.0005$; Wilcoxon). Values in parentheses indicate the numbers of cells, and error bars indicate SEM. Other measures of whole-field luminance change were investigated but were no better at predicting responses.

\section{Spatial organization of early RFs}

These data established that dLGN cells are able to detect spatial structure in natural stimuli presented through unopened eyelids. To explore what underlies this, we used flashed square stimuli to investigate through-the-eyelid RF structure. Through-the-eyelid RFs were obtained for 19 cells from three animals aged P29-30 (see Materials and Methods) (Fig. 8). The position of the display monitor was adjusted for each cell. This maximized the area of RF that could be recorded but meant that the RF locations do not reflect absolute positions in visual space. Responses to flashed square stimuli, the corresponding RFs, and responses to structured and unstructured movies are shown for three example neurons in Figure 8. The majority of the cells were like the example shown in Figure 8, $A$ and $B$. They were strongly unimodal in that they displayed either reliable On or Off profiles but not both. Furthermore, the RFs of these cells, although large, appeared to be localized to discrete parts of visual space. A smaller fraction of cells recorded before eye opening were like the cell in Figure $8 C$ and responded robustly to both On and Off stimuli. The RFs for these cells could occupy even larger portions of the visual field, but like the unimodal cells, they also showed localized structure.

To demonstrate that through-theeyelid RFs are spatially organized, we compared the real RFs to randomized RFs and used a smoothness measure to calculate the correlation between each RF pixel and its neighboring pixels (see Materials and Methods). A RF without spatial structure would be expected to show low correlation between neighboring pixels and so would have a low smoothness value. As shown in Figure $8 D$, almost $80 \%$ (15 of 19 ) of the real RFs had higher smoothness values than their randomized counterparts, a difference that was highly statistically significant ( $p<0.0005$; Wilcoxon). These data demonstrate that through-the-eyelid RFs exhibit spatial organization, and this presumably underlies the ability of the cells to detect changes in local luminance in the natural movie stimuli (i.e., the structure sensitivity described in Fig. 7). Nevertheless, the eyelid does have a marked blurring effect. This is highlighted by the fact that for cells in which RF size could be estimated by fitting a Gaussian function (see Materials and Methods), through-theeyelid RFs radii (median, $33.6^{\circ} ; n=11$ ) were approximately twice the size of RFs recorded immediately after eye opening at P32-34 (median, $14.8^{\circ} ; n=12$ ) and even twice the size of RFs recorded at P19-21 after the eyelids were opened experimentally (median, $15.4^{\circ} ; n=16$ ). The radii of through-the-eyelid RFs were significantly larger than both the open eyelid populations $(p<0.01$ in each case; Dunn post test after Kruskal-Wallis nonparametric ANOVA).

The observation that some through-the-eyelid RFs showed evidence of both On and Off responses (such as the cell in Fig. 8C) prompted us to explore whether there are changes in the arrangement of On and Off inputs to dLGN cells during this early period of visual responsiveness. We used reverse correlation methods to compare RFs from the first few days of visual responsiveness (P19-21) with those recorded at eye opening (P32-34). Importantly, the eyelids were opened for these experiments to collect data from as much of the RF as possible (without scattering because of the eyelids) and to allow comparison across the two age groups. Example RFs from the two age groups are shown in Fig- 
ure 9, $A$ and $B$. As the first example cell in Figure $9 A$ shows, RFs at the onset of visual responsiveness can be well organized. This cell is clearly most responsive to dark stimuli (Off-dominated), and the responsive region of visual space is both localized and relatively symmetrical in shape. This type of RF appeared very similar to typical RFs recorded just after eye opening at P32-34, as reflected by the example cells in Figure $9 B$. Although some P19-21 cells were comparable with those recorded at eye opening, other RFs at the onset of visual responsiveness appeared to be less organized. Two such examples are the second and third cells in Figure 9A that were recorded at P19 and P21, respectively. As their histograms and RF profiles show, these cells responded to both On and Off stimuli, and their RFs were not as clearly localized in visual space.

To compare the RFs quantitatively, we applied two measures of On-Off activity (Akerman et al., 2002). The first measure, On-Off ratio, calculates the relative strength of On and Off activity across the entire RF, returning higher values when either On or Off activity is dominant. These data are plotted for P19-21 and P32-34 in Figure $9 C$. The populations were not significantly different at the $95 \%$ level $(p=0.08$; Mann-Whitney $U$ test), although there was a tendency for younger cells to show lower On-Off ratios than those from older animals; whereas only $11 \%$ ( 2 of 19 ) of P32-34 cells had On-Off ratios below $0.25,32 \%$ (8 of 25 ) of the P19-21 cells fell into this category. The median On-Off ratio at P19-21 was $0.45(n=25)$ compared with $0.6(n=19)$ at $\mathrm{P} 32-34$. The second measure of On-Off activity, On-Off separation, calculates the spatial relationship between On and Off inputs, returning higher values when they occupy separate parts of visual space (see Materials and Methods). These data are plotted separately for P19-21 and P32-34 in Figure 9D. Again, the populations were not significantly different at the 95\% level ( $p=0.07$; MannWhitney $U$ test), although there was a tendency for younger cells to show higher OnOff separation: the median On-Off separation at P19-21 was $0.29(n=25)$ compared with 0.2 at P32-34 $(n=19)$. Together, these data indicate that On and Off pathways in the retinogeniculate pathway may not be fully mature at the onset of vision. However, RFs around the onset of visual responsiveness do show organization in terms of On and Off inputs, and changes over the following 2 weeks seem to be relatively subtle.

\section{Discussion}

The current study tested how experience patterns neuronal activity in vivo during the first 2 weeks of vision. In the ferret, this period corresponds to when the eyelids have not yet opened, but

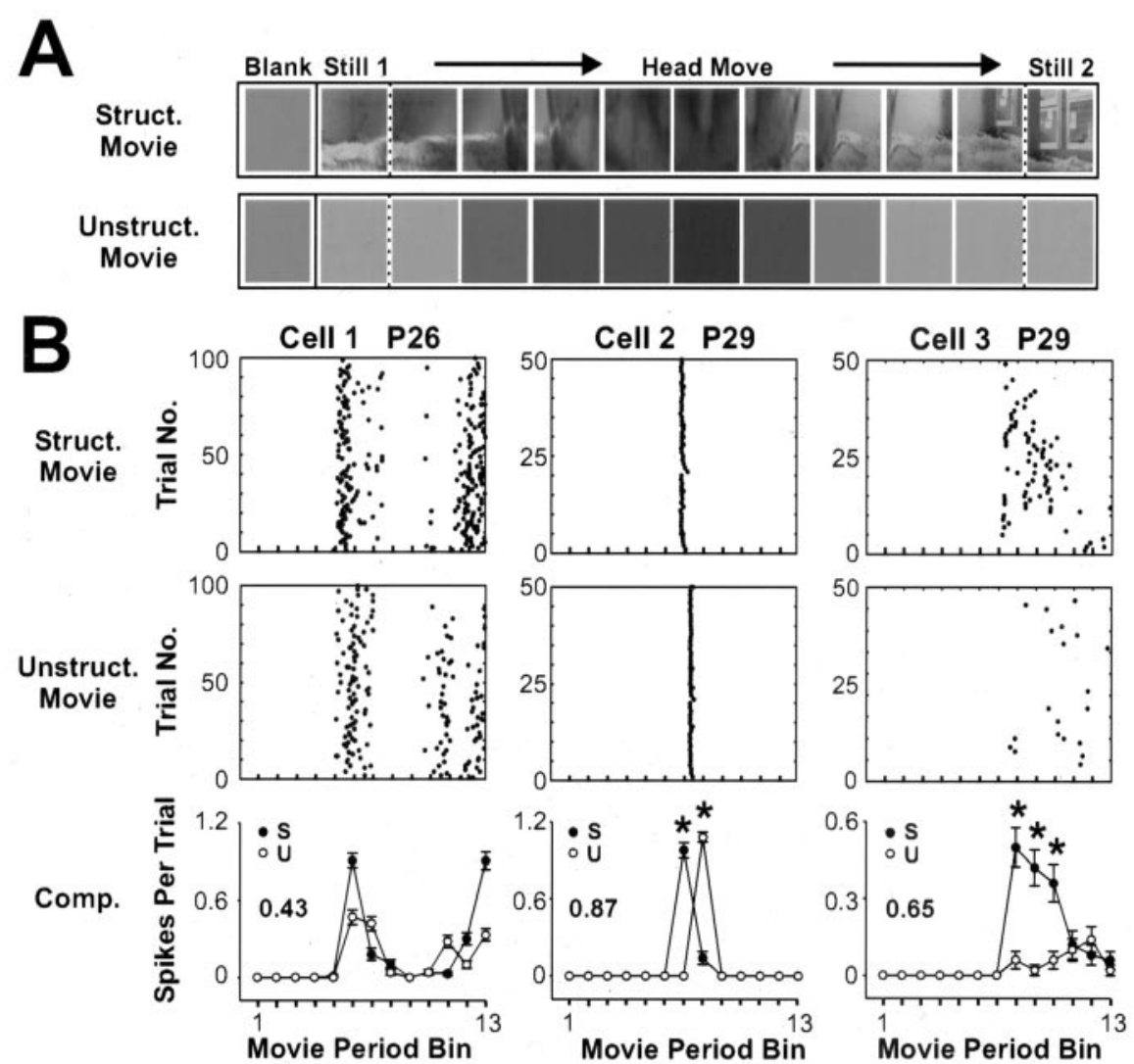

Figure 7. dLGN neurons can detect spatial structure in natural movies presented through closed eyelids. $A$, Structured movies were identical to the movie stimuli described above (Fig. 1). In unstructured movies, each frame was a structureless blank with luminance equal to the mean luminance of the corresponding frame of the structured movie. Three pairs of structured and unstructured movies were used. $B$, Rasters show responses of three cells to corresponding structured (top row) and unstructured movies (middle row). Each raster shows spiking activity during the movie period (see Materials and Methods and Fig. 1). Histograms (bottom row) compare structured (s) and unstructured ( $u$ ) movie responses. All three example cells were deemed structure sensitive. Cell 1, recorded at P26, exhibited a significantly higher firing rate during presentation of the entire structured movie and was, therefore, deemed structure sensitive (Mann-Whitney $p<0.05$ ). Cells 2 and 3, recorded at P29, showed significant differences in their responses to corresponding frames of the structured and unstructured movies $(p<0.05$; Dunn post test after Kruskal-Wallis nonparametric ANOVA). Of 44 neonatal dLGN neurons that responded to movies, 28 (64\%) were classified as structure sensitive. In the histograms, the asterisks denote bins in which responses differed between the two movie types ( $p<$ 0.05; Dunn post test after Kruskal-Wallis ANOVA), and the structure sensitivity index is stated on the left. $C$, The structure sensitivity index was used to compare the degree to which the responses of different cells were modulated by spatial structure in the movies (higher values indicate greater modulation by spatial structure; see Materials and Methods). The scatter plot shows that during this developmental window, there was a positive, but not statistically significant, correlation between structure sensitivity and age (Spearman $r=0.10 ; p=0.55$ ). Other conventions are as shown in Figure 2 .

responses can be recorded through the closed eyelids (see Results) (Krug et al., 2001), and visual experience at this stage can contribute to normal development (Akerman et al., 2002). First, we described the response patterns of single neurons in the primary visual pathway under conditions of natural visual stimulation. The responses of dLGN cells were shown to correlate with temporal changes in mean stimulus contrast, indicating that young cells detect whole-field luminance changes. Subsequent experiments showed that even through closed eyelids, responses 

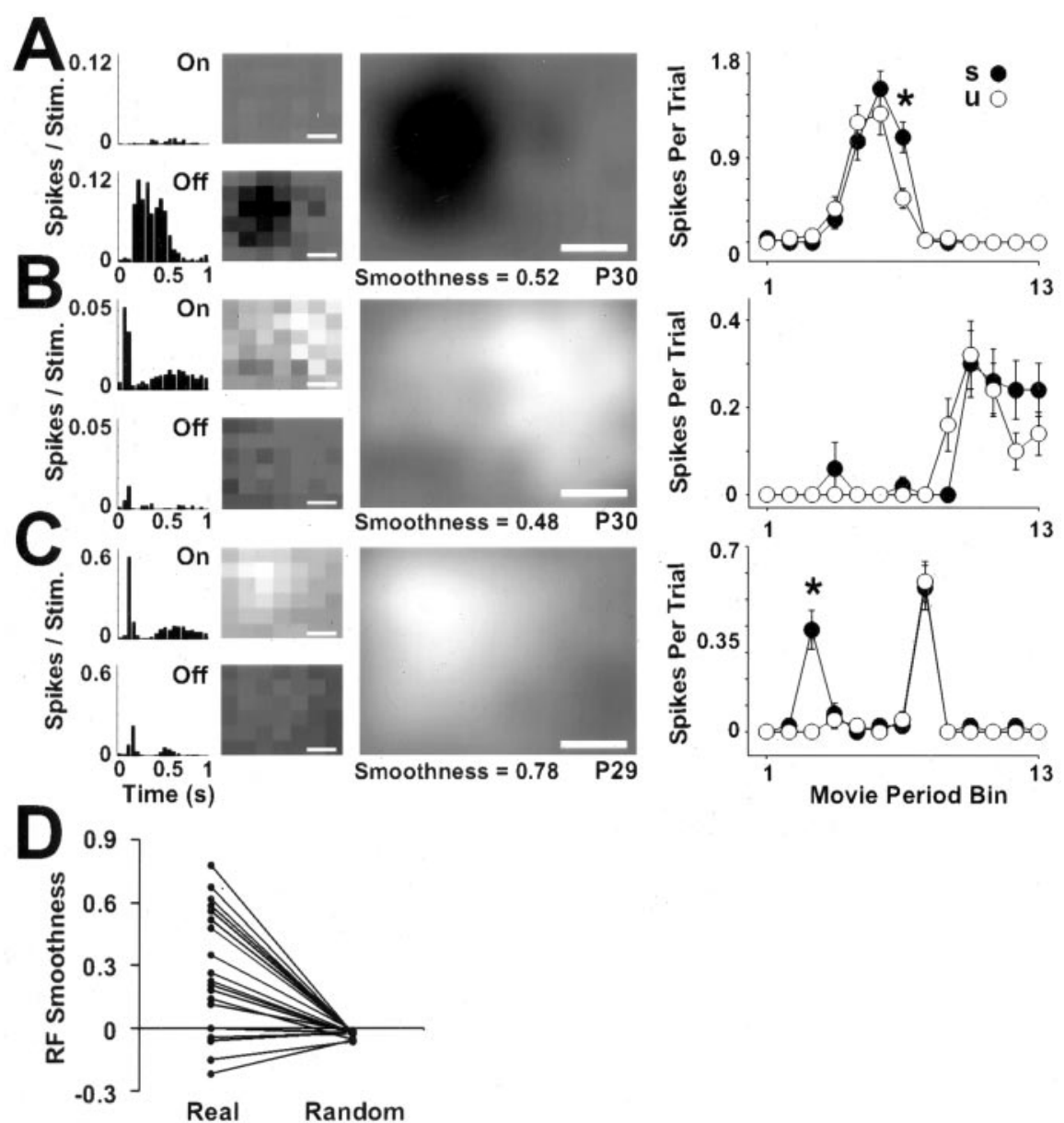

Figure 8. Through-the-eyelid RFs account for spatial structure detection during natural visual stimulation. To record from as much of the RF as possible, the display monitor was moved for each cell. $A-C$, Data are shown for three dLGN cells in which RFs were mapped through unopened eyelids (P29-30). A series of plots are presented for each cell. From left to right these are PSTHs of the mean spike rate in response to white (0n) and black (Off) flashed squares, raw RF profiles generated by reverse correlation (Jones and Palmer, 1987) from the spikes in the PSTHs, the combined (On minus Off) and smoothed RF map, and a line graph depicting the responses of the cell to structured and unstructured versions of a movie (same conventions as in Fig. 7). The age of recording and the RF smoothness are stated below each RF, and the scale bars represent $20^{\circ}$ of visual space. The cells in $A$ and $B$ were recorded on the same electrode penetration, within $500 \mu \mathrm{m}$ of one another. The cell in C was from a different animal. D, To quantify their spatial organization, we applied a measure of smoothness to the RFs and to randomized versions of the RFs (see Materials and Methods). Each line in the graph connects the real and random values for a single RF. According to this measure, $79 \%$ (15 of 19) of the real RFs were more organized than any of the randomized RFs.

of single neurons also contain information about spatial structure in natural scenes. Underlying this property of young geniculate neurons is the spatial localization and organization of their RFs. Responses to simple flash stimuli revealed that these early visual responses are also characterized by substantial variability between cells in their ability to detect stimuli of different duration and different intensity in a temporally precise manner.

\section{Relevance to the maturation of circuits}

The ferret has a relatively protracted period of postnatal development when it is visually responsive, but the eyelids have not yet opened. In species in which the eyes are open at birth (e.g., primates), light transmission through the closed eyelid may not be that relevant. However, for the species that are visually responsive before their eyes open postnatally [which includes mice (Tian and Copenhagen, 2003) and cats (Huttenlocher, 1967)], this may be the first source of visual experience. Perhaps more important for the current study is that the ferret is a model system for understanding the fundamental question of how neural activity shapes connections during development. In the period before eye opening, neuronal connections are changing throughout the ferret primary visual pathway, and many of these changes are thought to be modulated by activity. For instance, retinal afferents in the dLGN segregate first into eyespecific territories (Linden et al., 1981) and subsequently into $\mathrm{On}$ and Off zones (Hahm et al., 1999). In the period immediately after the onset of visual responsiveness, neuronal connections continue to change and refine in the ferret primary visual pathway. The maturation of On and Off pathways continues (Bodnarenko et al., 1999; Wang et al., 2001), and there are changes in the RF properties of visual neurons (Krug et al., 2001; White et al., 2001). What information could early visual responses provide that is relevant to these developing connections?

In vitro recordings have shown that during the period that On-center and Offcenter RGC afferents to the dLGN are segregating, Off-center RGCs are 3-4 times more spontaneously active than Oncenter RGCs (between P14 and P24) (Wong and Oakley, 1996; Myhr et al., 2001; Lee et al., 2002). This relative difference in spontaneous activity levels generates differences in correlations, which, when fed into a Hebbian model that incorporates inhibition and heterosynaptic competition, can simulate segregation of On and Off pathways (Lee et al., 2002). Our recordings are the first attempts to quantify the contribution of On and Off activity to ferret dLGN RFs at the onset of visual responsiveness, and we observed that single dLGN RFs at P19-21 are often dominated by a single polarity that can be localized in space (Fig. 9). Assuming that the earliest visual recordings reflect the population of cells in the retinogeniculate pathway, this observation is consistent with the view that functional segregation of On-center and Off-center retinogeniculate pathways can occur before and, therefore, independently of vision.

Other data have suggested that the On and Off pathways in the retinogeniculate pathway are not fully mature by the onset of visual responsiveness but develop over a longer period of time, which includes the period before eye opening (Bodnarenko et al., 1999; Wang et al., 2001; Tian and Copenhagen, 2003). Physiological and anatomical data show that at this stage the dendrites of a significant fraction of ferret RGCs are not completely stratified into either the On or Off sublamina of the inner plexiform layer (Wang et al., 2001). However, even before eye opening in the ferret, On-dominated and Off-dominated cell activity is strongly patterned under natural viewing conditions, with cells of the same type showing correlated activity but cells of different type showing anticorrelated activity (Akerman et al., 2002). This was also evident in the current study in which the activity of Ondominated cells was found to correlate with increases in mean 

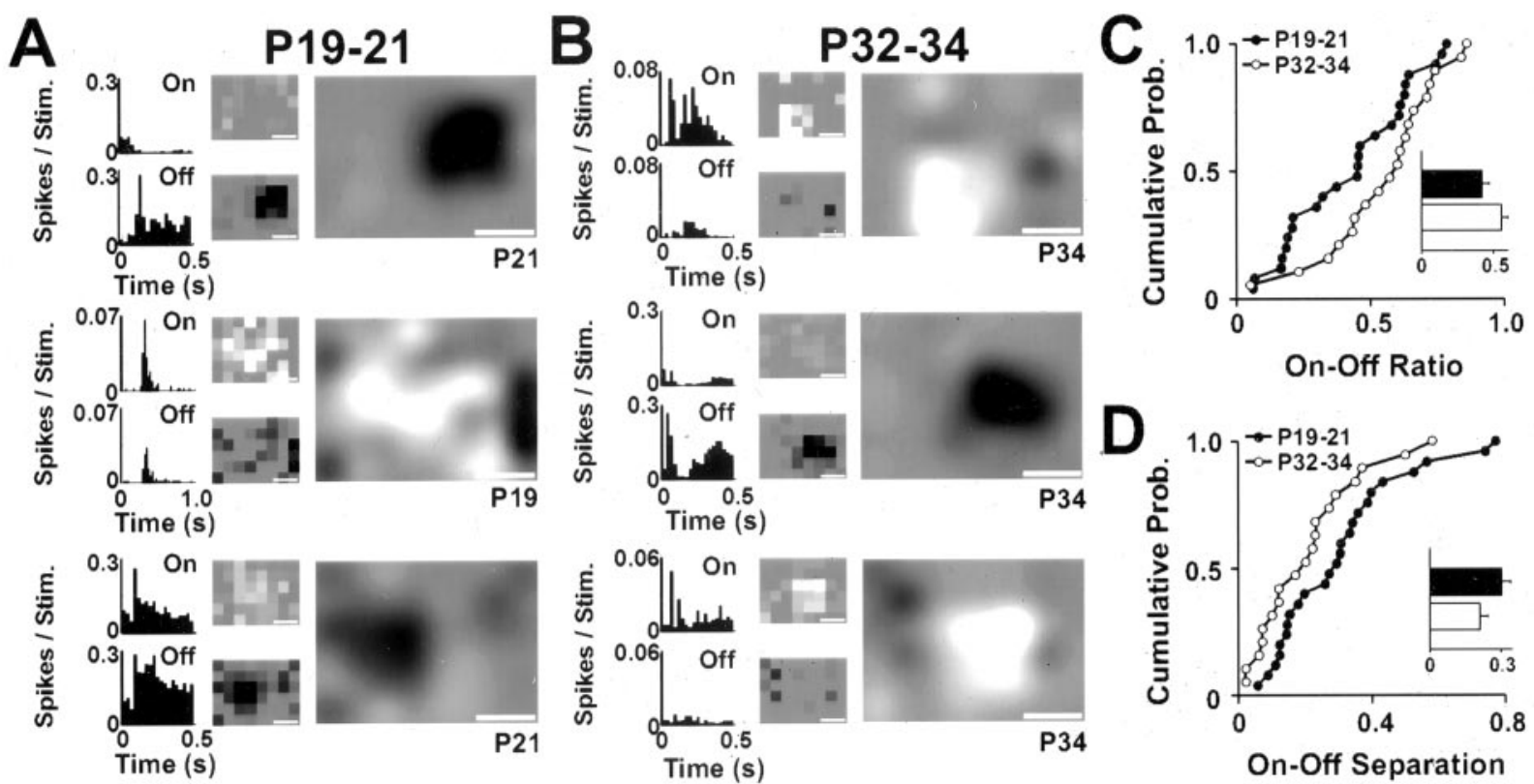

Figure 9. On and Off activity during the period of early visual responsivity. A, RFs of three dLGN neurons recorded during the first few days of visual responsiveness (P19-21). The eyelids were opened for these experiments to collect data from as much of the RF as possible (without scattering attributable to the eyelid) and to allow comparison with RFs recorded at the time of eye opening. $B$, RFs of three dLGN neurons recorded 2 weeks later, just after natural eye opening (P32-34). C, D, Cumulative probability plots of On-0ff Ratio ( $C$ ) and On-0ff separation ( $D$ ) derived from the RFs of dLGN neurons in P19-21 and P32-34 animals. There was a tendency for older cells to be more dominated by either On or Off activity and to have 0n and 0ff maps that were more concentric, although neither value reached statistical significance at the $95 \%$ confidence level ( $p=0.08$ and $p=0.07$; Mann-Whitney $U$ test; $0 \mathrm{n}-0 \mathrm{ff}$ ratio and $0 \mathrm{n}-0 \mathrm{ff}$ separation, respectively). Insets show the mean values for the two age groups; all other conventions are as shown in Figure 8.

luminance, whereas Off-dominated cell activity correlated with decreases in mean luminance (Fig. 6). Adopting a Hebbian-based view, this visually patterned activity would be well suited to segregate the two pathways by strengthening connections between cells of the same type at the same time as weakening connections between cells of different type. Consistent with this idea, dark rearing before eye opening affects the normal development of On and Off responses in the ferret dLGN (Akerman et al., 2002). As part of the current study, we compared RFs at the onset of visual responsiveness with those at eye opening (Fig. 9). These data did not indicate dramatic rearrangements in On and Off pathways over this period but, like previous recordings from the retina (Wang et al., 2001), suggest that On and Off may not be fully mature at the onset of visual responsiveness. It remains to be shown exactly which features of spontaneous and visually evoked activity contribute to the emergence and maintenance of $\mathrm{On}$ and Off pathways as development progresses.

The current study is the first to show that, at these early stages, dLGN cells are sensitive to spatial structure in natural scenes (Fig. 7) and have spatially localized RFs (Fig. 8). In principle, this spatial information could play a role in activity-dependent refinement of topography or in shaping RF properties. For instance, modeling data have shown that cortical simple cell RFs develop when same-cell correlations (e.g., between On cells) are stronger at small spatial separations and opposite-cell correlations (i.e., between On and Off cells) are stronger at larger separations (Miller, 1994; Miller et al., 1999). A prerequisite for such spatially relevant correlations is that the RFs of individual neurons are spatially localized, and our data demonstrate that this can be true for young dLGN neurons, even while the eyelids are closed. Our data do not test correlations across groups of neurons or test whether the spatial information is functionally relevant, but the large size of young RFs (Tavazoie and Reid, 2000) and the fact that they are blurred further by the closed eyelids (see Re- sults) suggests that activity-dependent correlations would occur over a large spatial scale during the first 2 weeks of vision in the ferret. Indeed, it is possible that early visual responses could actually degrade the refinement of retinotopic connections rather than actually improve it. To further investigate this problem, it will be interesting to record from multiple neurons simultaneously during periods of natural stimulation and periods of spontaneous activity.

\section{Constraints on response patterning during development}

Optical blurring properties of the eyelid, or even of the eye itself (Bonds and Freeman, 1978; Derrington, 1979), represent spatial constraints on how neural activity in the developing visual system is patterned by experience. Factors that influence the temporal response properties of neurons should also influence the patterning of activity during periods of sensory experience. For instance, variability in response times across geniculate cells will affect whether they are able to cooperate to activate a common postsynaptic cortical cell. A series of experiments using simple flash stimuli enabled us to explore systematically the temporal limits of early responses. dLGN cells at the onset of visual responsiveness had particularly long response latencies and showed substantial trial-to-trial variability in when they responded (Fig. 2). Even within the first 2 weeks of vision, we found strong developmental trends such that responses in older cells had both shorter latencies and were more temporally precise. Eye opening may contribute to the developmental decrease in latency. However, there was a significant trend in response latency even when the analysis was restricted to the period before eye opening, which was not attributable to changes in the eyelids (see Results). Therefore, it seems more likely that the first 2 weeks of vision represent the earliest period in the continuing maturation of visual response properties: from the onset of visual responsiveness in the ferret, latencies decrease during the period the eyes are closed and continue to do 
so after eye opening (Tavazoie and Reid, 2000). Furthermore, temporal response properties and stimulus sensitivity seem to follow a parallel course of development (Figs. 2-4), indicating that common factors may underlie these changes.

A major determinant of the early temporal properties is likely to be the maturation of phototransduction machinery. It is known that electrically evoked responses in the developing thalamus can be at least an order of magnitude faster than visually evoked responses (Hohnke et al., 2000), whereas visual responses in the retina display poor temporal resolution (Tootle, 1993). Furthermore, the onset and maturation of visual responsiveness is consistent with ultrastructural studies of the retina and the profile of photoreceptor protein expression (Greiner and Weidman, 1981; Johnson et al., 2001). Other factors influencing the temporal patterning could be myelination of the optic nerve (Schoppmann, 1985) and the temporal characteristics of synaptic transmission, although, at least for glutamatergic transmission at the retinogeniculate synapse, the largest changes seem to occur after eye opening (Ramoa and Prusky, 1997; Hohnke et al., 2000).

\section{Relevance to mechanisms of developmental plasticity}

Mechanisms underlying developmental plasticity are believed to be sensitive to the timing of spikes. The relative times of presynaptic inputs define how they summate to cause spikes in postsynaptic cells, and the timing of spikes in the postsynaptic cell can define a "temporal window" that, depending on the relative time of presynaptic activity, will lead to the potentiation or depression of inputs (Markram et al., 1997; Zhang et al., 1998; Feldman, 2000). If these timing-dependent plasticity mechanisms are operating in the ferret visual system, one prediction is that the variability in spike times, and how this changes with development, will be important.

The analysis of On and Off activity during early visual experience showed that developmentally relevant correlations exist at a timescale of $\sim 100 \mathrm{msec}$ (Akerman et al., 2002). However, at the ages we studied, only $25 \%$ of our developing dLGN neurons have precision values of $\leq 20 \mathrm{msec}$ (Fig. 2). Therefore, assuming that jitter is not systematically covarying across neurons, it seems unlikely that convergent inputs could regularly satisfy a narrow integration window (such as within $20 \mathrm{msec}$ ), as has been proposed for spike time-dependent synaptic potentiation (Zhang et al., 1998; Feldman 2000). Interestingly, experiments in the mammalian somatosensory cortex have shown that the temporal windows for synaptic potentiation and depression are not symmetrical, with depression occurring when spikes precede synaptic activation within a longer time window $(\sim 100 \mathrm{msec})$ (Feldman 2000). If such a temporally asymmetric mechanism operates during early vision, one would predict that temporal variability in early stimulus-evoked activity would favor the depression of inputs.

Alternatively, plasticity mechanisms with different temporal requirements might also operate during early development. Indeed, an information-theoretic study of spontaneous retinal waves during ferret development concluded that an optimal mechanism for retinotopic refinement would be sensitive to information over relatively coarse time scales (Butts and Rokshar, 2001). To take this further, developmental changes in the temporal fidelity of activity patterns may be reflected in the scale of refinements in connections. In other words, immature activity patterns that enforce temporally "loose" correlations may only be suitable for guiding relatively gross activity-dependent refinements. In contrast, temporally precise responses in the more mature visual system (i.e., after the first 2 weeks of vision) may be better suited for fine-scale refinements such as building RFs in higher visual areas.

\section{References}

Akerman CJ, Smyth D, Thompson ID (2002) Visual experience before eyeopening and the development of the retinogeniculate pathway. Neuron 36:869-879.

Bodnarenko SR, Yeung G, Thomas L, McCarthy M (1999) The development of retinal ganglion cell dendritic stratification in ferrets. NeuroReport 10:2955-2959.

Bonds AB, Freeman RD (1978) Development of optical quality in the kitten eye. Vision Res 18:391-398.

Butts DA, Rokhsar DS (2001) The information content of spontaneous retinal waves. J Neurosci 21:961-973.

Chapman B, Stryker MP (1993) Development of orientation selectivity in ferret visual cortex and effects of deprivation. J Neurosci 13:5251-5262.

Chiu C, Weliky M (2001) Spontaneous activity in developing ferret visual cortex in vivo. J Neurosci 21:8906-8914.

Chiu C, Weliky M (2002) Relationship of correlated spontaneous activity to functional ocular dominance columns in the developing visual cortex. Neuron 35:1123-1134.

Crowley JC, Katz LC (1999) Development of ocular dominance columns in the absence of retinal input. Nat Neurosci 2:1125-1130.

Derrington A (1979) Direct measurement of image quality in the kitten's eye. J Physiol 295:16P-17P.

Engert F, Tao HW, Zhang LI, Poo MM (2002) Moving visual stimuli rapidly induce direction sensitivity of developing tectal neurons. Nature 419:470-475.

Feldman DE (2000) Timing-based LTP and LTD at vertical inputs to layer II/III pyramidal cells in rat barrel cortex. Neuron 27:45-56.

Feller MB, Butts DA, Aaron HL, Rokhsar DS, Shatz CJ (1997) Dynamic processes shape spatiotemporal properties of retinal waves. Neuron 19:293-306.

Froemke RC, Dan Y (2002) Spike-timing-dependent synaptic modification induced by natural spike trains. Nature 416:433-438.

Greiner JV, Weidman TA (1981) Histogenesis of the ferret retina. Exp Eye Res 33:315-332.

Grubb MS, Thompson ID (2003) Quantitative characterisation of visual response properties in the mouse dorsal lateral geniculate nucleus. J Neurophysiol 90:3594-3607.

Guido W, Sherman SM (1998) Response latencies of cells in the cat's lateral geniculate nucleus are less variable during burst than tonic firing. Vis Neurosci 15:231-237.

Hahm JO, Cramer KS, Sur M (1999) Pattern formation by retinal afferents in the ferret lateral geniculate nucleus: developmental segregation and the role of N-methyl-D-aspartate receptors. J Comp Neurol 411:327-345.

Hohnke CD, Oray S, Sur M (2000) Activity-dependent patterning of retinogeniculate axons proceeds with a constant contribution from AMPA and NMDA receptors. J Neurosci 20:8051-8060.

Hubel DH, Wiesel TN (1965) Binocular interaction in striate cortex of kittens reared with artificial squint. J Neurophysiol 28:1041-1059.

Huberman AD, Wang GY, Liets LC, Collins OA, Chapman B, Chalupa LM (2003) Eye-specific retinogeniculate segregation independent of normal neuronal activity. Science 300:994-998.

Huttenlocher PR (1967) Development of cortical neuronal activity in the neonatal cat. Exp Neurol 17:247-262.

Jenison RL, Reale RA, Hind JE, Brugge JF (1998) Modeling of auditory spatial receptive fields with spherical approximation functions. J Neurophysiol 80:2645-2656.

Johnson PT, Williams RR, Reese BE (2001) Developmental patterns of protein expression in photoreceptors implicate distinct environmental versus cell-intrinsic mechanisms. Vis Neurosci 18:157-168.

Jones JP, Palmer LA (1987) The two-dimensional spatial structure of simple receptive fields in cat striate cortex. J Neurophysiol 58:1187-1211.

Katz LC, Shatz CJ (1996) Synaptic activity and the construction of cortical circuits. Science 274:1133-1138.

Krug K, Akerman CJ, Thompson ID (2001) Seeing with closed eyes: Responses of neurons in neonatal cortex and thalamus to patterned visual stimulation through the naturally closed eyelids. J Neurophysiol 85:1436-1443.

Lee CW, Eglen SJ, Wong RO (2002) Segregation of ON and OFF retino- 
geniculate connectivity directed by patterned spontaneous activity. J Neurophysiol 88:2311-2321.

Linden DC, Guillery RW, Cucchiaro J (1981) The dorsal lateral geniculate nucleus of the normal ferret and its postnatal development. J Comp Neurol 203:189-211.

Mainen ZF, Sejnowski TJ (1995) Reliability of spike timing in neocortical neurons. Science 268:1503-1506.

Markram H, Lubke J, Frotscher M, Sakmann B (1997) Regulation of synaptic efficacy by coincidence of postsynaptic APs and EPSPs. Science 275:213-215.

Meister M, Wong RO, Baylor DA, Shatz CJ (1991) Synchronous bursts of action potentials in ganglion cells of the developing mammalian retina. Science 252:939-943.

Miller KD (1994) A model for the development of simple cell receptive fields and the ordered arrangement of orientation columns through activitydependent competition between ON- and OFF-center inputs. J Neurosci 14:409-441.

Miller KD, Erwin E, Kayser A (1999) Is the development of orientation selectivity instructed by activity? J Neurobiol 41:44-57.

Myhr KL, Lukasiewicz PD, Wong RO (2001) Mechanisms underlying developmental changes in the firing patterns of ON and OFF retinal ganglion cells during refinement of their central projections. J Neurosci 21:8664-8671.

Ramoa AS, Prusky G (1997) Retinal activity regulates developmental switches in functional properties and ifenprodil sensitivity of NMDA receptors in the lateral geniculate nucleus. Brain Res Dev Brain Res 101:165-175.

Ruthazer ES, Akerman CJ, Cline HT (2003) Control of axon branch dynamics by correlated activity in vivo. Science 301:66-70.

Schoppmann A (1985) A developmental study of retinal afferents and visual responses in the cat pretectum. Exp Brain Res 60:350-362.
Sengpiel F, Stawinski P, Bonhoeffer T (1999) Influence of experience on orientation maps in cat visual cortex. Nat Neurosci 2:727-732.

Shouval H, Intrator N, Cooper LN (1997) BCM network develops orientation selectivity and ocular dominance in natural scene environment. $\mathrm{Vi}$ sion Res 37:3339-3342.

Stent GS (1971) A physiological mechanism for Hebb's postulate of learning. Proc Natl Acad Sci USA 70:997-1001.

Tavazoie SF, Reid RC (2000) Diverse receptive fields in the lateral geniculate nucleus during thalamocortical development. Nat Neurosci 3:608-616.

Tian N, Copenhagen DR (2003) Visual stimulation is required for refinement of ON and OFF pathways in postnatal retina. Neuron 39:85-96.

Tootle JS (1993) Early postnatal development of visual function in ganglion cells of the cat retina. J Neurophysiol 69:1645-1660.

Wang G-Y, Liets LC, Chalupa LM (2001) Unique functional properties of On and Off pathways in developing retina. J Neurosci 21:4310-4317.

Weliky M, Katz LC (1997) Disruption of orientation tuning in visual cortex by artificially correlated neuronal activity. Nature 386:680-685.

Weliky M, Katz LC (1999) Correlational structure of spontaneous neuronal activity in the developing lateral geniculate nucleus in vivo. Science 285:599-604.

White LE, Coppola DM, Fitzpatrick D (2001) The contribution of sensory experience to the maturation of orientation selectivity in ferret visual cortex. Nature 411:1049-1052.

Wong RO, Oakley DM (1996) Changing patterns of spontaneous bursting activity of on and off retinal ganglion cells during development. Neuron 16:1087-1095.

Zhang LI, Tao HW, Holt CE, Harris WA, Poo M (1998) A critical window for cooperation and competition among developing retinotectal synapses. Nature 395:37-44. 